\title{
Unraveling the mechanism of NO ligand photoisomerism by time-resolved infrared spectroscopy
}

\author{
Geoffrey Gallé ${ }^{\mathrm{a}}$, Matthieu Nicoul ${ }^{\mathrm{b}}$, Theo Woike ${ }^{\mathrm{c}}$, Dominik Schaniel ${ }^{\mathrm{d}}$, Eric Freysz ${ }^{\mathrm{a}, *}$ \\ ${ }^{a}$ Université de Bordeaux, CNRS UMR 5798, LOMA, 351 cours de la Libération, 33405 Talence, France \\ ${ }^{\mathrm{b}}$ I. Physikalisches Institut, Universität zu Köln, Zülpicherstr. 77, 50937 Köln, Germany \\ ${ }^{\mathrm{c}}$ Institut für Strukturphysik, TU Dresden, Zellescher Weg 16, 01062 Dresden, Germany \\ d CRM2, UMR-CNRS 7036, Université de Lorraine, BP 70239, Bvd des Aiguillettes, 54506 Vandoeuvre, France
}

\section{A B S T R A C T}

UV Vis and infrared femtosecond spectroscopy makes it possible to reveal all different steps of photochemical reactions after the electronic excitation The electronic relaxations are observed in the UV Vis spectral range whereas the nuclear motions are monitored in the infrared spectral range. We used femtosecond time resolved infrared spectroscopy to demonstrate the photoisomerization of the NO ligand photoinduced by a visible femtosecond pulse in a $\mathrm{Na}_{2}\left[\mathrm{Fe}(\mathrm{CN})_{5} \mathrm{NO}\right] \cdot 2 \mathrm{H}_{2} \mathrm{O}$ single crystal occurs in about $350 \mathrm{fs}$. The analysis of data makes it possible to unravel the mechanism leading to the photoiso merization of the NO ligand.

\section{Introduction}

Light induced ligand isomerization first described by Jørgensen and Werner about a century ago [1 3 ] is a special case of photo chemistry. Upon optical electronic excitation a structural rear rangement of one or several ligands towards a metastable configuration is induced [4 7]. It can even lead to an exchange of atoms between neighboring ligands [8]. In order to understand such isomerization phenomena all processes following the elec tronic excitation need to be investigated which can be achieved by time resolved spectroscopy on a femtosecond time scale [9]. The ultrafast study of electronic, vibrational and structural changes reveals the correlation between the modified electron density and the structural response of matter. Consider an electronic transition that excites a molecule from the ground state (GS) into an anti bonding excited state (ES). Such a complex in a transition state can undergo a nuclear motion e.g., the rotation of a ligand. The ultrafast electronic excitation is followed by a slower nuclear re sponse on the fs ps time scale $[10,11]$. According to the internal conversion the nuclear motion, e.g. rotation of the ligand, starts in a highly excited vibrational state of ES and in the absence of luminescence will cross over into highly excited vibrational rotational states of the novel geometry. The excess energy in this potential will be dissipated during the thermalization via vibra tional rotational states towards the ground state of the novel geometry [12]. If this novel geometry is metastable with sufficient lifetime, the structure can be determined by neutron or $\mathrm{X}$ ray

\footnotetext{
* Corresponding author. Fax: +33 540006970 .

E-mail address: e.freysz@cpmoh.u-bordeaux1.fr (E. Freysz).
}

diffraction [13 15]. Using UV Vis and infrared femtosecond spec troscopy all different steps of the photochemical reactions after the electronic excitation can be detected: the electronic relaxations are observed in the UV Vis spectral range and the nuclear motions are monitored in the infrared spectral range.

A typical example for such ultrafast photochemical processes is the reversible photoinduced linkage isomerization of the nitrosyl ligand in coordination compounds $\mathrm{M}$ NO $[6,13]$ with $\mathrm{M}$ a transi tion metal. We study here the prototypic case of the $\left[\mathrm{Fe}(\mathrm{CN})_{5} \mathrm{NO}\right]^{2}$ anion. As shown in Figure 1 the ground state (GS) is characterized by an almost linear Fe $\mathrm{N} \mathrm{O}$ coordination. Irradiation with light in the blue green spectral range (e.g. $\lambda \sim 400500 \mathrm{~nm}$ ) induces a charge transfer transition from the non bonding $3 d_{x y}$ orbital to the unoccupied anti bonding $\pi^{*}(\mathrm{NO})$ orbital. Thereby, the system changes the symmetry from a ${ }^{1} \mathrm{~A}_{1}$ state to a ${ }^{1} \mathrm{E}$ twofold degenerate state. According to the Jahn Teller theorem the degeneracy of the ${ }^{1} \mathrm{E}$ state will be lifted. As a consequence the doubly degenerate deformational mode $\delta(\mathrm{Fe} \mathrm{N} \mathrm{O})=666 \mathrm{~cm}^{1}$ can induce a rotation of the NO ligand [16 18]. The rotation of about $90^{\circ}$ yields the side on configuration of $\mathrm{Fe}<{ }^{\mathrm{N}}$ (metastable state MS2) while a rotation of $180^{\circ}$ results in the isonitrosyl configuration $\mathrm{Fe} O \mathrm{~N}$ (metastable state MS1). The structures of GS and of the metastable states MS1 and MS2 are known from X ray and neutron diffraction measurements at low temperatures in the static regime [19 21]. The lifetime of both states below 200 and $150 \mathrm{~K}$, respectively, is nearly infinite due to the activation energies of $0.68 \mathrm{eV}$ (MS1) and $0.43 \mathrm{eV}$ (MS2) [22]. Both metastable states are lying about $1 \mathrm{eV}$ above the ground state (see Figure 1) [23]. The transition from the excited state ${ }^{1} \mathrm{E}$ (ES) towards MS2 occurs radiationless with a time constant of $300 \mathrm{fs}$ [24]. MS2 has a lifetime of about $300 \mathrm{~ns}$ 


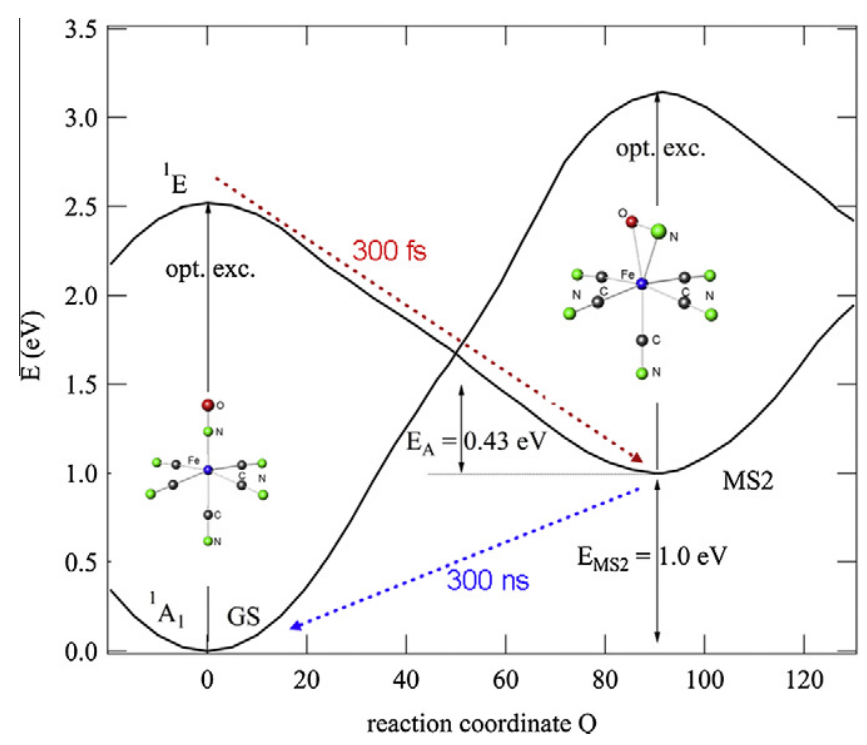

Figure 1. Potential scheme illustrating the NO linkage isomerisation process inferred from time-resolved visible spectroscopy [14].

at room temperature [25]. According to these experimental results in the visible spectral range, a potential scheme as shown in Figure 1 with crossing at the saddle point between GS and MS2 at $1.43 \mathrm{eV}$ was proposed [22]. Recently the competition between $\mathrm{NO}$ release and $\mathrm{NO}$ isomerisation in $\left[\mathrm{Fe}(\mathrm{CN})_{5} \mathrm{NO}\right]^{2}$ in solution was studied using time resolved ps infrared spectroscopy [26]. In agreement with the previous study [24] it was concluded that the isomers are formed on a subpicosecond timescale. However, a detailed knowledge about the relaxation processes in ES and the transition process from ES into MS1 or MS2 is still lacking, which is connected with the question where and when the $\mathrm{N} \mathrm{O}$ rotation starts. In order to shine more light on this relaxation from the excited ${ }^{1} \mathrm{E}$ state into the metastable states MS1 and MS2, we studied the rotation of the $\mathrm{NO}$ in $\mathrm{Na}_{2}\left[\mathrm{Fe}(\mathrm{CN})_{5} \mathrm{NO}\right] 2 \mathrm{H}_{2} \mathrm{O}$ (SNP, space group Pnnm) single crystals using polarized femtosecond infrared spectroscopy. In this manner the rotation of the NO ligand and the vibrational relaxation processes starting from the electroni cally excited state ES and ending in the metastable minimum of MS1 or MS2 can be observed. Infrared spectroscopy is especially suited for this purpose as GS, MS1, and MS2 can be clearly distin guished by the different $v(\mathrm{NO})$ stretching vibration frequencies at 1948,1835 and $1664 \mathrm{~cm}^{1}$, respectively. It yields an unambiguous identification of the corresponding relaxation pathways [26]. The use of single crystalline material has the advantage that we do not have to care about a possible solvent response which might mask the actual phenomena searched for [26]. Moreover in single crystals of SNP competing photochemical reactions like NO release are marginal when exciting in the visible spectral range.

\section{Experimental set-up}

The experimental set up is based on a $1 \mathrm{kHz}$ Ti:Sapphire regenerative amplifier system producing $50 \mathrm{fs}, 4.5 \mathrm{~mJ}$ laser pulses centered at $800 \mathrm{~nm}$ (Coherent Legend USP). The output of the laser system was split in two $1 \mathrm{~mJ}$ laser pulses which pumped two Optical Parametric Amplifiers (OPA, Model TOPAS from Light Conversion). One of these OPA was equipped with a Degenerate Frequency Generation (DFG) module for the generation of light in the spectral range of $200010.000 \mathrm{~nm}$. The maximum output en ergy is about $5 \mu \mathrm{J}$ in the $40006000 \mathrm{~nm}$ spectral range. The probe pulse energy used in our experiment is at least ten times lower. The pump beam generated by the second OPA centred at $\lambda \sim 500 \mathrm{~nm}$ and of energy of about $9 \mu \mathrm{J}$ was focused in the sample by a $10 \mathrm{~cm}$ ZnSe lens. The non collinear probe beam is focused within the sample using the same lens. The pump and probe beams overlap in the sample. We adjusted the lens so that within the sample the probe beam is more tightly focused than the pump beam. The sample used is a $270 \mu \mathrm{m}$ thick single crystal ( $b$ cut) of $\mathrm{Na}_{2}\left[\mathrm{Fe}(\mathrm{CN})_{5} \mathrm{NO}\right] 2 \mathrm{H}_{2} \mathrm{O}$. SNP crystallizes in the orthorhombic space group Pnnm. The polarization of the pump beam is parallel to the $c$ axis while the probe polarization is parallel to the $a$ axis. All measurements were performed at room temperature. Prior to the experiments, we measured the pump and probe pulse widths using an autocorrelation and a cross correlation (mixing the probe beam with the $50 \mathrm{fs}$ pulses centred at $800 \mathrm{~nm}$ in a nonlinear $\mathrm{LiNbO}_{3}$ crys tal) technique, yielding $120 \pm 10 \mathrm{fs}$ and $220 \pm 50$ fs for the pump and probe pulses, respectively. To measure the transient absorp tion signal at a given time delay $\tau$ between the pump and the probe, we used two $\mathrm{HgCdTe}$ detectors, placed before and after the sample, that measure the initial $\left(I_{r}\right)$ and the transmitted $\left(I_{t}(\tau)\right)$ intensity of the probe beam respectively. The detectors were cooled at liquid nitrogen temperature. The time delay $\tau$ between the probe and pump pulses was controlled using a motorized translation stage that has a spatial resolution of $1.5 \mu \mathrm{m}$. Hence our set up makes it possible to achieve a temporal resolution of \pm 10 fs. By computing the logarithm of $I_{r} / I_{t}(\tau)$, we determined the absorption signal $\mathrm{s}(\tau)=\alpha \times d=n \times \sigma \times d$ with $\alpha$ as absorption coefficient, $d$ thickness of the sample, $n$ number density of the occupied state and $\sigma$ as the cross section. The differential transient absorption signal $\Delta \mathrm{OD}(\tau)$, at a given delay $\tau$, was then computed by subtracting the absorption signal measured with and without the pump pulse. $\left[\mathrm{OD}=\log \left(I_{0} / I(\mathrm{t})\right)=\log [\exp (\alpha d)]=\alpha \times d \times\right.$ $\log (\mathrm{e})=n \sigma d \times 0.4343]$. For that purpose the pump beam was chopped at $40 \mathrm{~Hz}$. For our measurements, the typical measurement error was better than $10{ }^{3}$ optical density (OD). The overtones are measured with an absorption spectrometer in the spectral range of
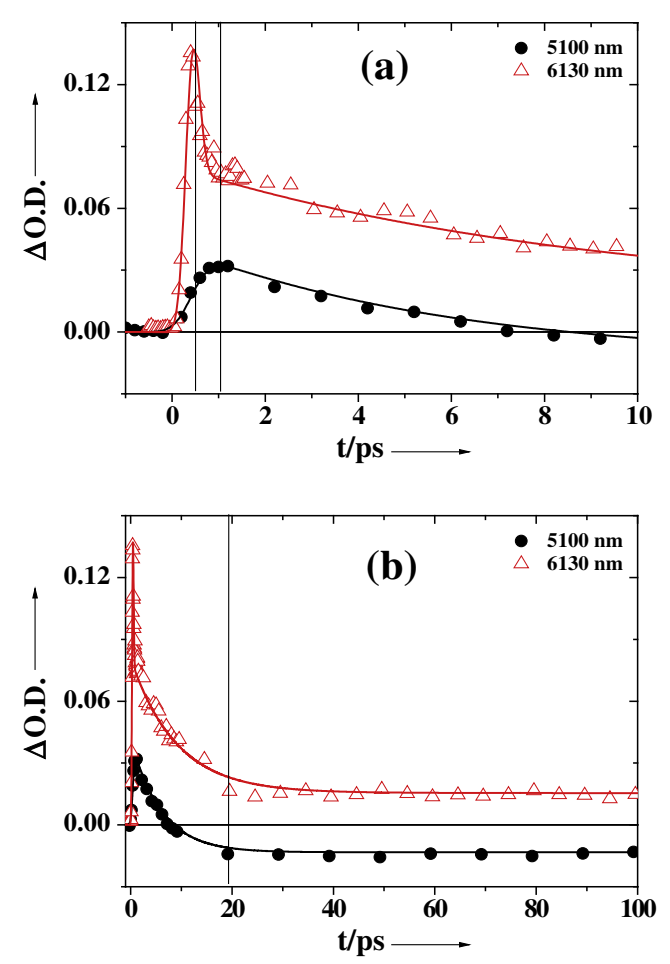

Figure 2. Temporal evolution of the differential transient absorption $\Delta \mathrm{OD}$ after excitation with $500 \mathrm{~nm}$ probed at the two wavelengths of the NO stretching vibration in GS $(5100 \mathrm{~nm})$ and MS2 $(6130 \mathrm{~nm})$. The solid lines are fittings of the data. 
$3300700 \mathrm{~nm}$ with a resolution of $6 \mathrm{~cm}{ }^{1}$. The polarization was chosen along all crystallographic axes to find clearly the total sym metric NO stretching modes. They are forbidden for light parallel to the $c$ axis. MS1 and MS2 are detected with a cryostat at $T=80 \mathrm{~K}$. The measured overtones are for GS at $T=296 \mathrm{~K}$ in $\mathrm{cm}^{1}$ : 1948 , 3872, 5769, 7639, 9483, 11.301. For MS2 at $T=80 \mathrm{~K}: 1664,3296$, 4890, 6454. For MS1 at $T=80 \mathrm{~K}: 1835,3637,5407,7147$.

Figure 2 shows the evolution of the differential transient absorption signal $\triangle \mathrm{OD}$ measured at the probe wavelengths $5100 \mathrm{~nm}\left(1961 \mathrm{~cm}^{1}\right)(\mathrm{GS})$, and $6130 \mathrm{~nm}\left(1631 \mathrm{~cm}^{1}\right)(\mathrm{MS} 2)$ after excitation with $500 \mathrm{~nm}$. At long timescales (Figure 2a) beyond 20 ps we observe constant values of $\Delta \mathrm{OD}$ that remain up to $1 \mathrm{~ns}$. At $6130 \mathrm{~nm}$ a positive differential transient absorption $\triangle \mathrm{OD} \sim 0.02$ is observed, indicating the population of the MS2 state. At $5100 \mathrm{~nm}$ a negative $\triangle O D \sim 0.015$ is observed corresponding to the bleach of the GS. Thus for timescales above 20 ps we conclude that the MS2 state is populated at the expense of the GS. Consider now the temporal evolution in the range $010 \mathrm{ps}$ (Figure $2 \mathrm{~b}$ ). In the first $340 \mathrm{fs}$ the signal is dominated by the cross correlation of pump and probe pulse inside the SNP crystal, so that no conclusion about molecular processes inside this time window can be drawn. At $340 \mathrm{fs}$ we observe a strong positive $\Delta \mathrm{OD} \sim 0.14$ at $6130 \mathrm{~nm}$, which then relaxes with two time constants of $300 \mathrm{fs}$ and $9 \mathrm{ps}$ before reaching the constant value at $20 \mathrm{ps}$. At $5100 \mathrm{~nm}$ the $\Delta O D$ rises after $340 \mathrm{fs}$ and reaches a maximum of $\Delta \mathrm{OD} \sim 0.03$ after about 1.2 ps with a time constant of $300 \mathrm{fs}$ before relaxing with a time constant of 6 ps towards its constant negative value at 20 ps.

Figure 3 shows the evolution of the differential transient absorption signal $\triangle O D$ measured after excitation with $500 \mathrm{~nm}$ at the probe wavelength $5460 \mathrm{~nm}\left(1835 \mathrm{~cm}^{1}\right)$ corresponding to the vibration frequency of the MS1 state. Since at the long time scale (i.e. beyond $20 \mathrm{ps}$ ), $\Delta \mathrm{OD} \sim 0$ these data clearly indicate that the MS1 state is not populated. However, one could clearly notice that upon excitation of the sample a transient absorption is recorded. This transient absorption basically follows the trend recorded for MS2 state. It increases during the pump excitation and then relaxes with long constant times of 9 ps. We noticed that we could improve the fitting of our data considering the relaxation was proceeding with two relaxations time of 6 and 9 ps. The weight of these two contributions is $10 \%$ and $90 \%$, respectively.

We can thus distinguish three phases in the temporal evolution:

(i) An immediate response within the experimental resolution of $340 \mathrm{fs}$ for $6130 \mathrm{~nm}$ and $5460 \mathrm{~nm}$.

(ii) A fast relaxation for $6130 \mathrm{~nm}$ as well as a fast increase for $5100 \mathrm{~nm}$ of the order of $300 \mathrm{fs}$.

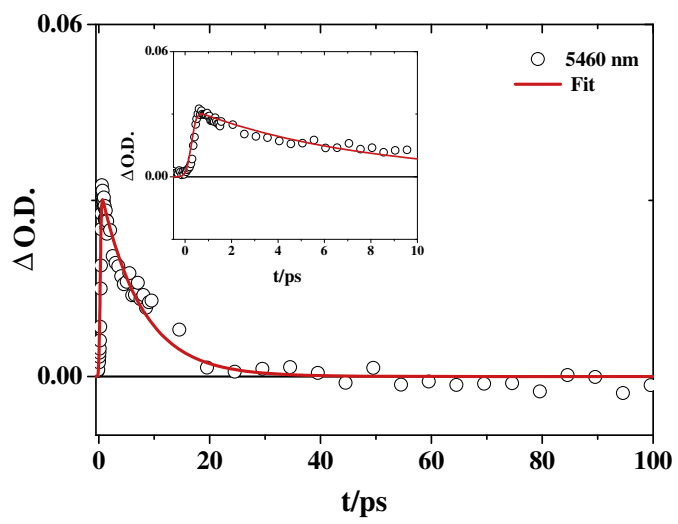

Figure 3. Temporal evolution of the differential transient absorption $\Delta \mathrm{OD}$ after excitation with $500 \mathrm{~nm}$ probed at the wavelengths of the NO stretching vibration in MS1 $(5460 \mathrm{~nm})$. The solid line is a fitting of the data. (iii) A long time relaxation of the order of $9 \mathrm{ps}$ for the probe wavelength centered at $6130 \mathrm{~nm}$, of the order of $6 \mathrm{ps}$ for the probe wavelengths centered at $5100 \mathrm{~nm}$ and and 6 and $9 \mathrm{ps}$ for the probe wavelengths centered at $5460 \mathrm{~nm}$.

\section{Discussion}

These observations allow for drawing a detailed picture of the processes leading to the photoisomerization (see Figure 3). In the first step the excitation from the GS to the excited state ES occurs, initiated by the $3 d x y \rightarrow \pi^{*}$ (NO) HOMO LUMO transition with an energy distance of $19762 \mathrm{~cm}{ }^{1}(2.45 \mathrm{eV}, 506 \mathrm{~nm})$ at $T=296 \mathrm{~K}$ when detected parallel to the $a$ axis [28]. An important observa tion is that immediately after the illumination (pump pulse of $120 \mathrm{fs}$ duration) with $500 \mathrm{~nm}$ light the NO vibration can be observed as absorption at $6130 \mathrm{~nm}\left(1631 \mathrm{~cm}{ }^{1}\right)$. Thus the distance between two neighboring $v(\mathrm{NO})$ vibrational levels in ES are close to those in MS2, which are known to be at $1664 \mathrm{~cm}^{1}$ from low temperature $(T<150 \mathrm{~K})$ static infrared spectroscopy [27]. We can thus conclude that in this novel electronic configuration the Fe $\mathrm{N}$ O is kinked with respect to the linear GS configuration which is in agreement with DFT calculations for the transition from GS to MS2 [16 18], which propose an Fe N O angle of $3050^{\circ}$ in ES. The increase of the absorption at $5460 \mathrm{~nm}$ is also a good indication that indeed some of the molecules in the ES state have a kick angle lar ger than $45^{\circ}$. Indeed in such a case, one could consider that the vibrational excited state can be decomposed into the three $v(\mathrm{NO})$ vibrational levels associated to the MS1, MS2 and GS states. This explains the immediate observation of a strong absorption at 6130 and $5460 \mathrm{~nm}$ in ES. For the other probe wavelength $5100 \mathrm{~nm}\left(1961 \mathrm{~cm}^{1}\right)$ an absorptive signal can be detected only after the depopulation of the ES and the subsequent occupation of GS. Therefore, the decay time (300 fs) of ES corresponds exactly to the increase ( $300 \mathrm{fs}$ ) of MS2.

The first $\mathrm{Fe} \mathrm{N} \mathrm{O}$ bending following the electronic excitation corresponds to an intermediate excited state. The molecule can now follow two different paths: either it returns back to the linear GS configuration or it continues its rotation towards the MS2 side on configuration. Let us stress that the absence of an absorption at $5460 \mathrm{~nm}$ on the longer time scale discards the relaxation channel towards the MS1 state. The observation of a decrease of absorption at $6130 \mathrm{~nm}$ as well as an increase at $5100 \mathrm{~nm}$ with the same time constant of 300 fs illustrates these steps in the photoisomerization. The excited intermediate state ES is depopulated towards GS and MS2 mainly by internal conversion. In Figure 4 these transitions

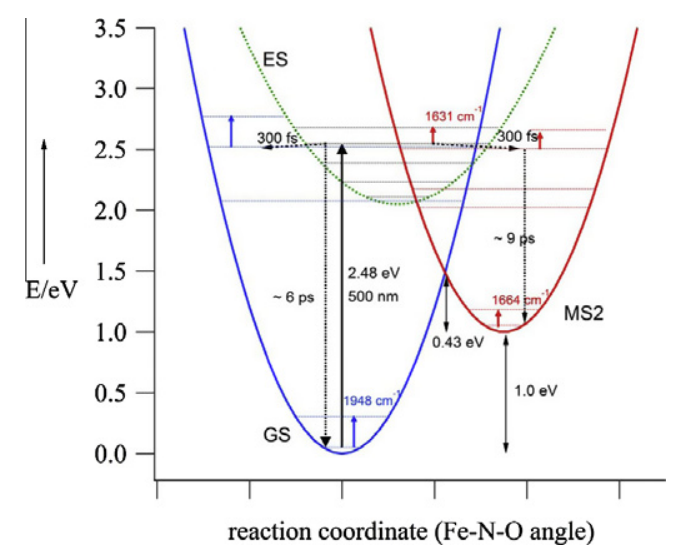

Figure 4. Two dimensional cut through the potential hyperspace illustrating the photoisomerization process via internal conversion in SNP. The $\mathrm{v}(\mathrm{NO})$ frequencies indicated for GS $\left(1948 \mathrm{~cm}^{1}\right.$ at RT) and MS2 $\left(1664 \mathrm{~cm}^{1}\right.$ at $\left.80 \mathrm{~K}\right)$ are obtained from static infrared spectroscopy [18]. 
out of ES are illustrated for ES, GS and MS2 within a potential scheme. The cut through the hyper space is chosen in such a way that at fixed bond lengths changes the reaction coordinate corre sponds to the Fe $\mathrm{N} \mathrm{O}$ angle. By the excitation with $20.000 \mathrm{~cm}^{1}$ an $v(\mathrm{NO})$ stretching vibration in ES is occupied from which the measured transition $\left(1631 \mathrm{~cm}^{1}\right)$ to higher vibrational levels occurs. In order to facilitate an internal conversion the GS should exhibit a vibrational level of $v(\mathrm{NO})$ at around $20.000 \mathrm{~cm}{ }^{1}$. In order to explore the positions of the $v(\mathrm{NO})$ overtones in GS and MS2 we have performed polarized absorption spectroscopy on thick SNP single crystals. For GS we found $v(\mathrm{NO})$ overtones up to $n=6$ $\left(11.301 \mathrm{~cm}{ }^{1}\right.$ ) at $T=23{ }^{\circ} \mathrm{C}$ (see experimental details). From the positions we could extrapolate with a polynom of order 4 the positions of the $v(\mathrm{NO})$ overtones up to $n=10$ yielding $\mathrm{E}(11)=$ $19.274 \mathrm{~cm}^{1}$. The zero point energy is $\mathrm{E}(0)=984 \mathrm{~cm}{ }^{1}$.

For MS2 the $v(\mathrm{NO})$ overtones were measured at $T=80 \mathrm{~K}$ since MS2 decays around $150 \mathrm{~K}$ due to the activation energy of $0.43 \mathrm{eV}$. Energetically the MS2 minimum is $1 \mathrm{eV}$ above GS. The measurements yielded results up to the fourth overtones (see experimental details) and the extrapolation up to $n=7$ yields $E(7)=11.612 \mathrm{~cm}{ }^{1}$. Adding the energetic position of the MS2 minimum $8066 \mathrm{~cm}^{1}(1 \mathrm{eV})$ yields $19678 \mathrm{~cm}^{1}$, which is very close to the excitation energy of $20000 \mathrm{~cm}^{1}$. The zero point energy in MS2 is $\mathrm{E}(0)=842 \mathrm{~cm}^{1}$. Thus energetically an internal conversion from ES to GS and MS2 is possible. Furthermore all the states have the same multiplicity $1 \mathrm{~A}$ and no luminescence was found during the decay of MS1 or MS2 in the spectral range $2001200 \mathrm{~nm}$ up to now. Moreover MS2 is diamagnetic and thus the occupation of a triplet state in ES is highly improbable as it would require a double spin flip, one from GS to ES and one from ES to MS. The steady sequence of overtones in MS2 and MS1 up to $0.8 \mathrm{eV}$ further hints to a potential crossing between GS/ MS2 and GS/MS1. For the case of an avoided crossing we would expect a discontinuity in the sequence of overtones.

Due to the fact that immediately after pulsed excitation at $500 \mathrm{~nm}$ we observe absorption at both 5460 and $6130 \mathrm{~nm}$, the geometry of ES is very likely a kinked NO configuration which is agreement with DFT calculations that propose an $\mathrm{Fe} \mathrm{N} O$ angle in the range of $4050^{\circ}$ as proposed by DFT calculations [16 18]. The minimum of the energetic position of ES is unknown, but we know that the crossing of GS and MS2 is at $1.43 \mathrm{eV} \mathrm{[22]} \mathrm{and} \mathrm{so}$ $1.06 \mathrm{eV}$ below the excitation with $2.49 \mathrm{eV}$. Then a relaxation from ES into GS and MS2 by occupation of highly excited NO vibrational levels that are energetically close to the NO vibrational levels of ES can occur within $\tau=300 \mathrm{fs}$. A precedent relaxation within ES would need more time and we therefore exclude this possibility. This fast decoupling of the ES towards MS2 or back to GS is in accordance with the results of the time resolved study in the visi ble range [24] where an occupation of MS2 within $300 \mathrm{fs}$ was found. Thus the rotation of the NO ligand both towards $90^{\circ}$ as well a back to $0^{\circ}$ occurs with $\tau=300$ fs. We can now specify that after this first fast relaxation the molecules are in a highly vibrationally excited state of the corresponding ground states (GS or MS2).

From this vibrationally excited states the thermalization of the molecules towards the corresponding minima in GS or MS2 occurs with time constants of $\tau=6 \mathrm{ps}$ (GS) and $\tau=9 \mathrm{ps}$ (MS2). From the knowledge of the extrapolated overtones of the $v(\mathrm{NO})$ stretching vibrations in GS (see experimental details) we can estimate that in GS the thermalization occurs starting from $20000 \mathrm{~cm}^{1}$ via 10 NO levels, such that for every transition about 600 fs are needed. The fact that the absorption at $5460 \mathrm{~nm}$ relaxes with the same con stants time as the one we recorded for the thermalization MS2 state indicates that it is mainly the release of the energy towards the molecular and lattice vibrations that accounts for the absorp tion at $5460 \mathrm{~nm}$ absorption. As this excess of energy is released, the molecule reaches its mimimum position within its potential well, this absorption at $5460 \mathrm{~nm}$ is cancelled out.
The picture we have elaborated can now be compared to ab initio calculations including excited states $[16,17,29,30]$. In these studies the GS potential is calculated as a function of the $\mathrm{Fe} \mathrm{N} \mathrm{O}$ angle. The energetic positions of the metastable minima and saddle points are in general calculated too high as compared to the exper iment. The doubly degenerate excited state 1E (ES) is considered Jahn Teller active and consequently upon optical excitation the symmetry of the system is spontaneously lowered resulting in two non degenerate states $1 \mathrm{~A}^{\prime \prime}$ and $1 \mathrm{~A}^{\prime}$. In [17] the geometrical optimization yields an eclipsed bent $\mathrm{Fe} \mathrm{N} \mathrm{O}$ geometry for the more stable $1 \mathrm{~A}^{\prime \prime}$ state with a Fe $\mathrm{N} \mathrm{O}$ angle of about $40^{\circ}$ and a Jahn Teller stabilization energy of about $0.33 \mathrm{eV} .1 \mathrm{~A}^{\prime \prime}$ is thus about $2.1 \mathrm{eV}$ above the GS. Deactivation from this relaxed excited state then occurs via a non radiative process or a conical intersection. Given that we excite with $2.5 \mathrm{eV}$ the system is $0.4 \mathrm{eV}$ above this relaxed excited state such that the vibrational excitation of the ex cited state $1 \mathrm{E}$ is limited to one or two levels $\left(0.4 \mathrm{eV} \sim 3226 \mathrm{~cm}^{1}\right)$ supposing that their distance is $1664 \mathrm{~cm}{ }^{1}$. This picture agrees well with the one illustrated in Figure 4. Notably the distance be tween the $v(\mathrm{NO})$ vibrations is much smaller in the $40^{\circ}$ configura tion compared to the $1948 \mathrm{~cm}^{1}$ of GS. Thus the decision whether GS, MS1 or MS2 is occupied is taken in ES. To this the potentials of GS, MS1 and MS2 must exhibit crossing points, so that the vibrationally excited states of GS, MS1 or MS2 can be occupied. It can be expected that this kind of photoisomerization scheme ap plies also to MS1 and to the other compounds of the ML5NO family as well as to similar photoswitchable ligands such as $\mathrm{N} 2, \mathrm{NO} 2, \mathrm{SO} 2$ etc., in which the multiplicity $1 \mathrm{~A}$ is conserved in all states and thus no intersystem crossing occurs.

\section{Conclusion}

In conclusion we observed the ultrafast photoisomerization in SNP using time resolved infrared spectroscopy. A complete description of the initial process for the NO photoisomerization could be deduced on the basis of internal conversion. The optical excitation leads to an excited state from which the rapid transition into MS2 and GS occurs within 300 fs due to vibronic coupling. The rotation of the NO ligand either to $90^{\circ}$ or back to $0^{\circ}$ proceeds with this time constant. After these fast $(\sim 300 \mathrm{fs})$ transitions the vibrational rotational thermalization to the GS and MS2 potential minima occurs within a few picoseconds.

\section{Acknowledgements}

Financial supports by the DFG (SCHA1550/1 1), National Research Agency (ANR 09 BLAN 0212) and Conseil Régional d'Aquitaine are gratefully acknowledged.

\section{References}

[1] S.M. Jørgensen, Z. Anorg. Allg. Chem. 5 (1894) 147.

[2] S.M. Jørgensen, Z. Anorg. Allg. Chem. 19 (1899) 109

[3] A. Werner, Ber. Deutsh. Chem. Ges. 40 (1907) 765.

[4] J. Burmeister, Coord. Chem. Rev. 3 (1968) 225.

[5] J. Burmeister, Coord. Chem. Rev. 105 (1990) 77.

[6] P. Coppens, I. Novozhilova, A. Kovalevsky, Chem. Rev. 102 (2002) 861

[7] Y. He, A. Gahlmann, J.S. Feenstra, S.T. Park, A. Zewail, Chem. Asian J. 1-2 (2006) 56.

[8] A.Y. Kovalevsky, G. King, K.A. Bagley, P. Coppens, Chem. Eur. J. 11 (2005) 7254

[9] F.C. De Schryver, S. De Feyter, G. Schweitzer, Femtochemistry, WILEY-VCH, Weinheim, 2001.

[10] K.R. Sawyer et al., J. Phys. Chem. A 112 (2008) 8505.

[11] I.M. Lane, D.A. King, Z.-P. Liu, H. Arnolds, Phys. Rev. Lett. 97 (2006) 186105.

[12] G.M. Sando, Q. Zhong, J.C. Owrutsky, J. Chem. Phys. 121 (2004) 2158.

[13] J. Schefer, D. Schaniel, V. Petricek, T. Woike, Z. Kristallogr. 4-5 (2008) 259.

[14] P. Coppens, Angew. Chem. Int. Ed. 48 (2009) 4280.

[15] M.R. Warren et al., Angew. Chem. Int. Ed. 48 (2009) 5711

[16] M. Atanasov, T. Schönherr, J. Mol. Struct. 592 (2002) 79. 
[17] M. Buchs, C.A. Daul, P.T. Manoharan, C.W. Schläpfer, Int. J. Quant. Chem. 91 (2003) 418.

[18] E. Coronado, S. Kloishner, O. Reu, B. Tsukerblat, Polyhedron (2003) 2527.

[19] M.D. Carducci, M.R. Pressprich, P. Coppens, J. Am. Chem. Soc. 119 (1997) 2669

[20] D. Schaniel, T. Woike, J. Schefer, V. Petricek, Phys. Rev. B 71 (2005) 174112.

[21] D. Schaniel, T. Woike, J. Schefer, V. Petricek, K.W. Krämer, H.U. Güdel, Phys. Rev. B 73 (2006) 174108.

[22] D. Schaniel, T. Woike, L. Tsankov, M. Imlau, Thermochim. Acta 429 (2005) 19.

[23] T. Woike, W. Krasser, H. Zöllner, W. Kirchner, S. Haussühl, Z. Phys. D 25 (1993) 351

[24] D. Schaniel, M. Nicoul, T. Woike, Phys. Chem. Chem. Phys. 12 (2010) 9029.
[25] D. Schaniel, Th. Woike, C. Merschjann, M. Imlau, Phys. Rev. B 72 (2005) 195119.

[26] M.S. Lynch, M. Cheng, B.E. Van Kuiken, M. Khalil, J. Am. Chem. Soc. 133 (2011) 5255.

[27] M.E. Chacon Villalba, J.A. Guida, E.L. Varetti, P.J. Aymonino, Inorg. Chem. 42 (2003) 2622.

[28] D. Schaniel, J. Schefer, B. Delley, M. Imlau, Th. Woike, Phys. Rev. B 66 (2002) 085103.

[29] B. Delley, J. Phys.: Cond. Mat. 22 (2010) 384208.

[30] B. Delley, J. Schefer, Th. Woike, J. Chem. Phys. 107 (1997) 10067. 